\title{
Is polaron effect important for resonant Raman scattering in self-assembled quantum dots?
}

\author{
M. I. Vasilevskiy ${ }^{*}$ and R. P. Miranda \\ Centro de Física, Universidade do Minho, 4710-057 Braga, Portugal
}

Received 29 June 2004, accepted 24 September 2004

Published online 15 February 2005

PACS 63.22.+m, 71.55.Gs, 78.30.-j

\begin{abstract}
While the diagonal (or intra-level) interaction of a confined exciton with optical phonons in selfassembled quantum dots (SAQD's) is rather weak, the non-diagonal one can lead to a considerable change of the exciton spectrum and the formation of a polaron. An impact of this effect on resonant inelastic light scattering is studied theoretically. The polaron spectrum is obtained by numerical diagonalisation of the exciton-phonon interaction Hamiltonian in a truncated Hilbert space of the non-interacting excitons and phonons. Based on this spectrum, the probability of the multi-phonon Raman scattering is calculated, which is compared to that obtained within the standard perturbation theory approach (where phonon emission and absorption are irreversible). It is shown that there are two major effects of the polaron formation: (i) the intensity of the two-phonon ( $2 \mathrm{LO}$ ) peak, relative to that of the fundamental $1 \mathrm{LO}$ one is strongly increased and (ii) the resonant behaviour of the 1 LO peak differs considerably from the perturbation theory predictions. With the correct theoretical interpretation, resonant Raman scattering in SAQD's opens the possibility of accessing the (renormalised) exciton spectrum and exciton-phonon coupling constants.
\end{abstract}

(ㄷ) 2005 WILEY-VCH Verlag GmbH \& Co. KGaA, Weinheim

\section{Introduction}

The electron-phonon interaction is important in semiconductors and, especially, in semiconductor nanostructures since it determines hot carrier relaxation, influences light absorption and emission processes and is responsible for Raman scattering. The intensity of this interaction is enhanced in quantum dots (QD's) and, owing to the discrete nature of the electron energy spectrum, it leads to multi-phonon processes and formation of a polaron [1]. As far as excitons are concerned, the importance of the polaron effect is less obvious, because the exciton-phonon interaction may be greatly reduced due to the compensation of the electron and hole charge densities in the dot. However, this compensation is only partial, and the diagonal (or intra-level) exciton-phonon interaction is still strong enough to produce a significant effect in spherical II-VI QD's [2, 3]. The non-diagonal interaction (involving two different exciton states) is not subject to the compensation effect and can be quite important even in III-V self-assembled QD's (SAQD's). Thus, the correct description of optical transitions requires a non-adiabatic and nonperturbative treatment of the many-body interactions taking place in these systems [1-3].

The purpose of the present work is to study the importance of the polaron effect (owing mostly to the non-diagonal exciton-phonon coupling) for the resonant Raman scattering (RRS) in SAQD's. RRS is known to be a powerful tool for obtaining information not only on phonons but also on the underlying electronic spectra [4]. Even though, there are rather few experimental studies of RRS in SAQD's so far. Information on phonon modes in the most intensively investigated SAQD system, InAs/GaAs, has been obtained by resonant photoluminescence [5] rather than by Raman scattering, although there are some

\footnotetext{
Corresponding author: e-mail: mikhail@fisica.uminho.pt
} 
published studies of SAQD's grown in other heteroepitaxial systems (see [6] and references therein). From the theoretical viewpoint, RRS in SAQD's was considered in Ref. [7] in the framework of the standard perturbation theory approach.

In this work, we use a general non-perturbative approach to the calculation of the multi-phonon Raman scattering cross-section. The basic idea is to consider the most relevant exciton states together with a small number of optical phonon modes and truncate the Hamiltonian matrix by including just a limited number of phonons allowed for each mode, large enough to guarantee that the result can be considered exact in the physically important region of energies. Such an approach has been shown to yield a very accurate solution for the polaron spectrum and stationary states of QD's [2]. Given the eigenvalues and eigenstates of the interacting exciton-phonon system, any physical quantity of interest can be obtained. In our previous work [8], such an approach has been applied to the calculation of the one-phonon Raman scattering probability in II-VI QD's. Here we extend it to multi-phonon RRS and apply to a typical InAs/GaAs SAQD.

\section{Formalism and model}

In a standard perturbation theory approach, valid when the exciton-phonon interaction is weak and/or if there is a continuum of exciton states, the $k$-phonon Raman scattering can be considered as a $(k+2)$-th order process where an exciton is created by absorption of an incident photon of frequency $\omega_{I}$, then it is successively scattered through the generation (for the Stokes component) of $k$ optical phonons and finally disappears by emitting a photon of a different frequency, $\omega_{S}=\omega_{I}-\sum_{v=1}^{N} r_{v} \omega_{v}$, where $r_{v}$ denotes the number of phonons of mode $v$ and frequency $\omega_{v}$ created in the process, subject to the condition $\sum_{v=1}^{N} r_{v}=k$. The scattering probability can be calculated using the Fermi Golden Rule and summing over all possible sets $\left\{r_{v}\right\}$. The details of such a calculation can be found in Ref. [9]. For parallel polarisations of the incident and scattered photons, the scattering probability is given by

$$
\begin{aligned}
& p=\frac{2 \pi}{\hbar}\left(\frac{e}{m_{0} \eta}\right)^{4}\left(\frac{2 \pi \hbar}{V}\right)^{2} \frac{n_{I}}{\omega_{I} \omega_{S}} \frac{1}{(k !)^{2}} \sum_{\left\{r_{v}\right\}}\left\{\prod_{v=1}^{N} \frac{\left(r_{V} !\right)^{3}\left(\hbar \omega_{v}\right)^{2 r_{v}}}{\left[1-\exp \left(-\frac{\hbar \omega_{v}}{k_{B} T}\right)\right]^{r_{v}}}\right. \\
& \times\left[\sum_{\left\{v_{k}\right\}}\left[\sum_{l_{1}, l_{2}, \ldots, l_{k+1}} \frac{p_{0 l_{1}} p_{0 l_{k+1}}^{*} \prod_{i=1}^{k} \beta_{l_{i} l_{i+1}}^{\left(v_{i}\right)}}{\prod_{i=0}^{k}\left(\hbar \omega_{I}-\sum_{j=1}^{i} \hbar \omega_{v_{j}}-E_{l_{i+1}}\right)}\right] \delta\left(\hbar \omega_{I}-\hbar \omega_{S}-E_{\left\{r_{v}\right\}}\right)\right\}
\end{aligned}
$$

where $e$ and $m_{0}$ are the free-electron charge and mass, respectively, $V$ denotes the scattering volume, $\eta$ is the refractive index for the incident and scattered frequencies, $p_{0 l_{i}}$ is the momentum matrix element between the exciton states $|0\rangle$ and $\left|l_{i}\right\rangle, E_{\left\{r_{v}\right\}}=\sum_{v=1}^{N} r_{v} \hbar \omega_{v}$ and $\beta_{l_{i} i_{i+1}}^{\left(v_{i}\right)}$ denotes the exciton-phonon interaction constants for the phonon mode $v_{i}$ expressed in units of $\hbar \omega_{v_{i}}$. The explicit form of the latter depends on the interaction mechanism, which will be specified below. Notice that the expression for $p$ derived in Ref. [9] is valid for $T=0$ while Eq. (1) is general.

In the polaron picture, the (Stokes) Raman scattering process consists in two transitions, one with absorption and the other with emission of a photon, which change the QD state from exciton vacuum plus $\left\{m_{v}\right\}$ phonons to exciton vacuum plus $\left\{m_{v}^{\prime}\right\}$ phonons, with $m_{v}^{\prime}=m_{v}+r_{v}$. The exciton-photon inter- 
action can still be treated perturbatively but the intermediate states are those of the polaron. The scattering probability is now given by

$$
p=\frac{2 \pi}{\hbar} \frac{1}{Z} \sum_{i, f}\left\{\exp \left(-\frac{E_{i}}{k_{\mathrm{B}} T}\right)\left|\sum_{\kappa} \frac{\left\langle f\left|\hat{H}_{e R}^{(S)}\right| \kappa\right\rangle\left\langle\kappa\left|\hat{H}_{e R}^{(I)}\right| i\right\rangle}{E_{\kappa}-E_{i}-\hbar \omega_{I}}\right|^{2} \delta\left(E_{f}-E_{i}-\hbar \omega_{I}\right)\right\}
$$

where an average over the initial states and a sum over the final ones have been included,

$$
Z=\sum_{\left\{m_{v}\right\}} \exp \left(-\frac{E_{\left\{m_{v}\right\}}}{k_{\mathrm{B}} T}\right)
$$

and $|\kappa\rangle=\left|\alpha ; n_{I}-1,0_{S}\right\rangle$ are the intermediate states, with $n_{I(S)}$ denoting the number of incident (scattered) photons. In this expression, $|\alpha\rangle$ denotes the $\alpha$-th exciton-polaron state of energy $E_{\alpha}$, which are to be found by diagonalising the exciton-phonon interaction Hamiltonian $[2,8]$.

It is convenient to express these states as linear combinations of the eigenstates of the uncoupled exciton-phonon system, $\left|l ;\left\{m_{v}\right\}\right\rangle$ :

$$
|\alpha\rangle=\sum_{l,\left\{m_{v}\right\}} c_{l,\left\{m_{v}\right\}}^{(\alpha)}\left|l ;\left\{m_{v}\right\}\right\rangle .
$$

As mentioned in the Introduction and explained in more detail in Refs. [2, 8], the numerical diagonalisation of the exciton-phonon Hamiltonian matrix provides the polaron energy spectrum and the expansion coefficients, $c_{l,\left\{m_{v}\right\}}^{(\alpha)}$.

The scattering probability can therefore be written as

$$
\begin{aligned}
p= & \frac{2 \pi}{\hbar}\left(\frac{e}{m_{0} \eta}\right)^{4}\left(\frac{2 \pi \hbar}{V}\right)^{2} \frac{n_{I}}{\omega_{I} \omega_{S}} \frac{1}{Z} \sum_{\left\{m_{v}\right\}} \exp \left(-\frac{E_{\left\{m_{v}\right\}}}{k_{\mathrm{B}} T}\right) \\
& \times \sum_{\left\{r_{v}\right\}}\left\{\left|\sum_{\alpha} \frac{\sum_{l, l^{\prime}} p_{0 l} p_{0 l^{\prime}}^{*}\left(c_{l,\left\{m_{v}\right\}}^{(\alpha)}\right)^{*} c_{l^{\prime},\left\{m_{v}\right\}^{\prime}}^{(\alpha)}}{E_{\alpha}-E_{\left\{m_{v}\right\}}-\hbar \omega_{I}}\right| \delta\left(\hbar \omega_{I}-\hbar \omega_{S}-E_{\left\{r_{v}\right\}}\right)\right\} .
\end{aligned}
$$

We have applied both the perturbative and polaron approaches to a model system representing an InAs/GaAs SAQD. In a typical InAs/GaAs dot, the ground exciton state, $|1\rangle$, is some $E_{1}=0.9-1 \mathrm{eV}$ above the exciton vacuum $(|0\rangle)$ level $[1,5,10]$. The first excited exciton state, $\left|2^{ \pm}\right\rangle$, is two times degenerate if the QD has a perfect axial symmetry and is separated by some $\Delta E=E_{2}-E_{1}=60-70 \mathrm{meV}$ from the ground state. The anisotropy in the growth plane can split this state, with the separation being of the order of $\delta E_{2}=7 \mathrm{meV}$ [1]. These three bare exciton states were included in the model. Both the ground and excited states are optically active. The momentum matrix element for the $|0\rangle \rightarrow|1\rangle$ transition is assumed to be two times large than that for $|0\rangle \rightarrow\left|2^{-}\right\rangle$and $|0\rangle \rightarrow\left|2^{+}\right\rangle$.

The most important phonon modes are InAs LO of the QDs $\left(\hbar \omega_{1}=32 \mathrm{meV}\right)$ and of the wetting layer $\left(\hbar \omega_{2}=30 \mathrm{meV}\right)$ [5]. Some authors also consider an interface InAs/GaAs optical phonon but we confined our model by just two InAs modes. The exciton-phonon interaction is supposed to be mostly of the Fröhlich type. Owing to the relatively low degree of ionicity of InAs and the compensation effect mentioned in the Introduction, the diagonal exciton-phonon coupling constants are supposed to be rather small, $\beta_{11} \approx \beta_{22} \leq 0.01$, for the mode 1 (QD mode). The coupling between the levels $|1\rangle$ and $\left|2^{ \pm}\right\rangle$is expected to be much stronger, because these exciton states are different mostly by the electron component. Thus, the non-diagonal exciton-phonon interaction constant, $\beta_{12}$, should be of the order of the electronphonon one. The value of 0.15 was used for the latter in Ref. [1] in order to fit the experimentally observed variation of the far-infrared absorption spectra with applied magnetic field. We shall assume that $\beta_{12} \sim 0.1-0.2$ for the mode 1 . For the wetting layer phonon mode all the coupling constants are assumed to be two times smaller. 

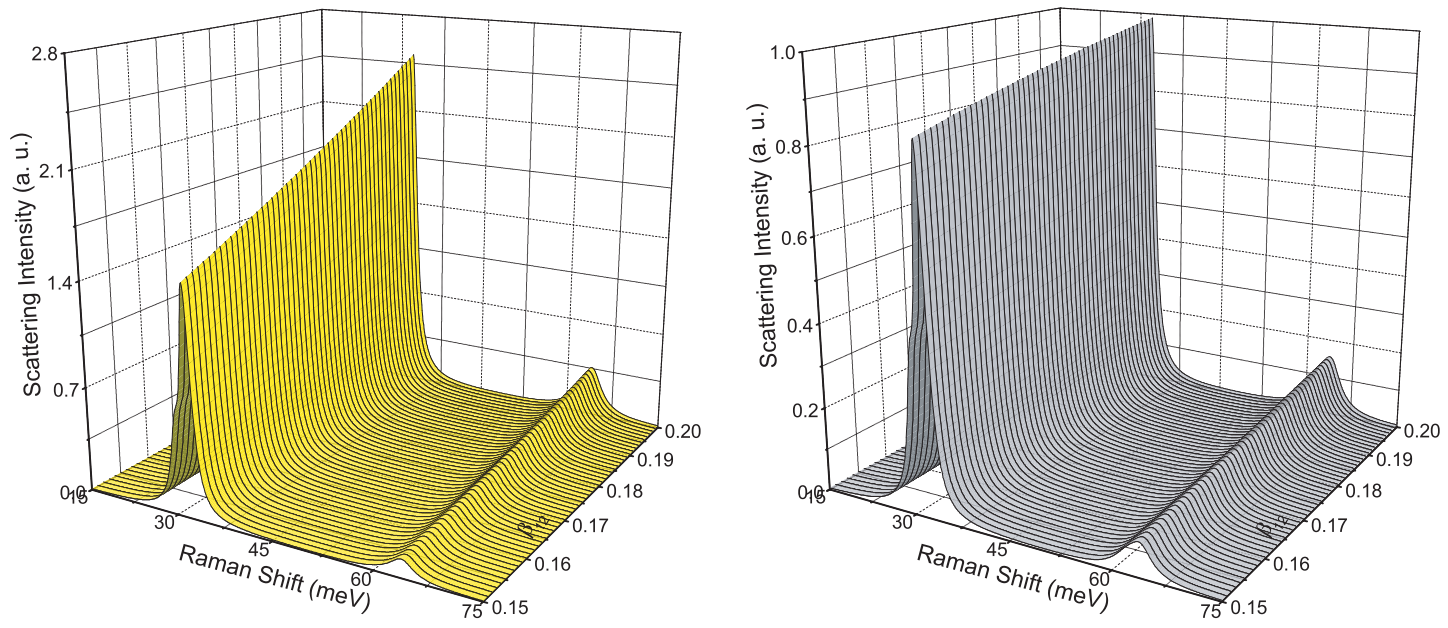

Fig. 1 Raman spectra (up to the second order) calculated at room temperature for different values of the inter-level coupling constant using the perturbative (a) and polaronic (b) approaches, assuming $\Delta E=70 \mathrm{meV}$ and $\delta E_{2}=0$.

\section{Results and discussion}

The RRS spectra calculated including the first and second order processes are presented in Fig. 1 for several values of the non-diagonal coupling constant $\beta_{12}$. The shape of the $1 \mathrm{LO}$ peak (determined by the relative contribution of two phonon modes included in the model) is somewhat different in the spectra calculated using the perturbation theory approximation and the polaron approach, but it is more noticeable the difference in the intensity of the second peak relative to the first one. We have checked that, in the limit of small $\beta_{12}$, when all $\beta_{i j}$ are below 0.01 , the spectra obtained using two approaches coincide. Thus, the observed enhancement of the relative intensity of the $2 \mathrm{LO}$ peak is merely a consequence of the polaron effect.

Figure 2 shows the excitation spectra for the first order Raman peak where the results calculated using two approaches also differ substantially. When the inter-level coupling is strong, the spectrum calculated using Eq. (3) (contrary to that obtained with Eq. (1)) shows characteristic features similar to those obtained in the electron spectral function and known as Rabi splitting [2]. They correspond to the exciton oscillating between its two states and (many times) emitting and absorbing a phonon.

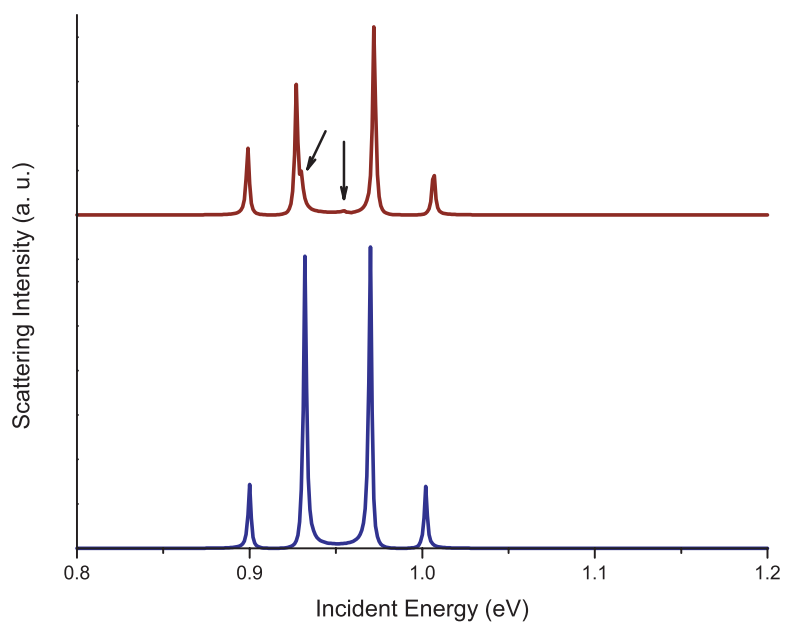

Fig. 2 Excitation spectra for the first order Raman peak calculated at $T=77 \mathrm{~K}$ using the perturbative (lower curve) and polaronic (upper curve) approaches, considering $\beta_{12}=0.2$, $\Delta E=70 \mathrm{meV}$ and $\delta E_{2}=0$. The arrows indicate small features related to the Rabi-type oscillations between two exciton levels. 
The importance of the polaron effect for the Raman spectra depends not only on the value of the exciton-phonon coupling but also on the inter-level distance, $\Delta E$, and increases when $\Delta E$ becomes smaller. Comparing to our previously calculated results [8] for CdSe QD's, where $\Delta E$ was of the order of $100 \mathrm{meV}$, the polaron effect manifests itself at much lower values of $\beta_{12}$. At the same time, we did not find any special behaviour near the $\Delta E=2 \hbar \omega_{1}$ resonance. Let us note also that the value of the upper level splitting $\left(\delta E_{2}\right)$ was found to produce little effect on the calculated spectra.

In conclusion, we proposed a non-perturbative approach to the calculation of the multi-phonon resonant Raman scattering probability in semiconductor QDs and applied it to a model InAs/GaAs SAQD. By comparing the results calculated using this theory with those obtained in the standard perturbative approach, it has been shown that the polaron effect does play an important role in the Raman scattering, even for materials like InAs with relatively small exciton-phonon coupling constants. Thus, Raman scattering and scattering excitation spectroscopy of SAQD's, if analysed beyond the standard perturbation theory approach, can be used for probing the exciton spectra (renormalised by the polaron effect) and evaluation of the exciton-phonon coupling constants.

Acknowledgements This work was supported by the Portuguese Foundation for Science and Technology (FCT) through projects POCTI/FIS/10128/98 and POCTI/CTM/39395/2001.

\section{References}

[1] S. Hameau et al., Phys. Rev. Lett. 83, 4152 (1999).

[2] M. I. Vasilevskiy, E. V. Anda, and S. S. Makler, Phys. Rev. B 69 (in press).

[3] V. M. Fomin et al., Phys. Rev. B 57, 2415 (1998).

[4] P. Y. Yu and M. Cardona, Fundamentals of Semiconductors: Physics and Materials Properties (Springer, Berlin 1996).

[5] N. N. Ledentsov et al., Semiconductors B 32, 343 (1998).

[6] D. A. Tenne et al., Phys. Rev. B 61, 13785 (2000).

[7] E. Menéndez-Proupin, C. Trallero-Guiner, and S. E. Ulloa, Phys. Rev. B 60, 16747 (1999).

[8] M. I. Vasilevskiy, R. P. Miranda, E. V. Anda, and S. S. Makler, Semicond. Sci. Technol. 19, S312 (2004).

[9] R. Rodríguez-Suárez, E. Menéndez-Proupin, C. Trallero-Guiner, and M. Cardona, Phys. Rev. B 62, 11006 (2000).

[10] N. V. Baidus et al., Semicond. Sci. Technol. 19, S468 (2004). 